Space Ops 2014, Pasadena, California

Abstract

\title{
Extravehicular Activity Asteroid Exploration and Sample Collection Capability
}

\author{
Zebulon Scoville, Stephanie Sipila, Jonathan Bowie
}

NASA Johnson Space Center, Houston, TX 77058

\begin{abstract}
NASA's Asteroid Redirect Crewed Mission (ARCM) is challenged with primary mission objectives of demonstrating deep space Extravehicular Activity (EVA) and tools, and obtaining asteroid samples to return to Earth for further study. Although the Modified Advanced Crew Escape Suit (MACES) is used for the EVAs, it has limited mobility which increases fatigue and decreases the crews' capability to perform EVA tasks. Furthermore, previous Shuttle and International Space Station (ISS) spacewalks have benefited from EVA interfaces which have been designed and manufactured on Earth. Rigid structurally mounted handrails, and tools with customized interfaces and restraints optimize EVA performance. For ARCM, some vehicle interfaces and tools can leverage heritage designs and experience. However, when the crew ventures onto an asteroid capture bag to explore the asteroid and collect rock samples, EVA complexity increases due to the uncertainty of the asteroid properties. The variability of rock size, shape and composition, as well as bunching of the fabric bag will complicate EVA translation, tool restraint and body stabilization. The unknown asteroid hardness and brittleness will complicate tool use. The rock surface will introduce added safety concerns for cut gloves and debris control.
\end{abstract}

Feasible solutions to meet ARCM EVA objectives were identified using experience gained during Apollo, Shuttle, and ISS EVAs, terrestrial mountaineering practices, NASA Extreme Environment Mission Operations (NEEMO) 16 mission, and during Neutral Buoyancy Laboratory testing in the MACES suit. The proposed concept utilizes expandable booms and integrated features of the asteroid capture bag to position and restrain the crew at the asteroid worksite. These methods enable the capability to perform both finesse, and high load tasks necessary to collect samples for scientific characterization of the asteroid.

This paper will explore the design trade space and options that were examined for EVA, the overall concept for the EVAs including translation paths and body restraint methods, potential tools used to extract the samples, design implications for the Asteroid Redirect Vehicle (ARV) for EVA, the results of early development testing of potential EVA tasks, and extensibility of the EVA architecture to NASA's exploration missions. 